\title{
La investigación sobre la comunicación en Francia. Tendencias y carencias
}

\author{
Thierry Lancien, Hélène Cardy, Janine Delatte, \\ Gilles Delavaud, Pascal Froissart, Anolga \\ Rodionof, Marie Thonon, Patricio TuPPer*
}

El presente artículo es el resultado de las ponencias presentadas durante el Coloquio "La comunicación en Europa, la búsqueda de las relaciones entre investigación y sociedad", en el que no se buscó establecer un panorama completo de la investigación en comunicación en Francia sino más bien, a partir de diferentes tipos de indicadores, intentar organizar una primera cartografía sobre el estado de la cuestión. De esta forma se presentan los trazos de una primera exploración: en lo referente a la disciplina en Francia, seguida del análisis de la situación de los organismos públicos relacionados con el tema, así como de las revistas y de los nuevos conceptos de la materia, concluyendo con un análisis sobre las listas de discusión sobre la investigación y la comunicación desarrolladas en Internet.
This article is the result of the papers presented in the conference "Communication in Europe, seeking relationships among research and society", which did not pretend to establish a whole overview about communication research in France but, from different kind of indicators, try to organize a first instance cartography about that subject. Thus, a first exploration outline is presented: conditions of this discipline in France; an analysis of the situation of public organizations related to the subject, as well as of the reviews and new concepts about that topic; it finishes with a discussion lists analysis on those research and communication practices which had been carry out in the Internet.

*Universidad de París VIII y Grupo de Estudio e investigación sobre los

Dispositivos y los Actores de la Comunicación (GRÉDAC). 


\section{LA HISTORIA}

\section{Patricio Tupper}

Dentro del plano institucional, el nacimiento de las ciencias de la información y de la comunicación (CIC) constituye la obra de algunos pioneros, entre quienes se encuentran Robert Escarpit, Jean Meyriat y Roland Barthes. A finales de los años sesenta, este "trío" (al que podríamos agregar a Abraham Moles, menos comprometido por los juegos institucionales) buscaba obtener un reconocimiento universitario dentro de las preocupaciones científicas de un cierto número de profesores que trabajaban en el campo de la información y, sobre todo, de estructurar disciplinariamente los nuevos programas de enseñanza ofrecidos por los IUT -y en algunos casos, en los primeros años de licenciatura - como el caso de Periodismo o de Formaciones sobre el libro, y los cursos técnicos de expresión y comunicación. Esta empresa buscaba como objetivo la creación de una nueva sección en el interior del Consejo Consultivo de las Universidades (CCU).

A finales de 1971, la Comisión Pedagógica Nacional (CPN) de los IUT, en la especialidad "Carreras de la información", presidida por Robert Escarpit, adoptó una moción que solicitaba a los CCU crear una sección que correspondiese a las "Ciencias de la información y de la comunicación", la de los doctorantes en esta especialidad y la de una comisión de cic en el comité nacional del CNRS. Esta moción fue avalada rápidamente por los consejos de administración de varias universidades.

Paralelamente a las gestiones universitarias, el presidente de la CPN, auxiliado por Jean Meyriat y Roland Barthes, llevaba a cabo una campaña de sensibilización ante los colegas que trabajaban en especialidades complementarias. El 25 de febrero de 1972, se propuso una reunión en la Casa de las Ciencias del Hombre (MSN, por sus siglas en francés) de París, en la que participaron 44 personas. Ahí se tomó la decisión de crear una disciplina, a la que se denominó Ciencias de la información y de la comunicación, luego de haber considerado otras denominaciones como: Ciencias de la representación o Ciencias de la significación. 
Jean Meyriat comentó durante una entrevista con Jean Devèze y Anne-Marie Laulan, con motivo de la celebración de los 20 años de la Sociedad Francesa de las Ciencias de la Información y la Comunicación (SFSIF, por sus siglas en francés), recordando ese debate: "A final de cuentas, el término de CIC, se conservó por razones de eficacia: la sensación prevaleció en el sentido de que el término más concreto de ‘información' precisa más al término vago de 'comunicación'. Este binomio permite, al mismo tiempo, servir a los intereses de diferentes grupos de distintos especialistas, sin perder una posición definitiva sobre la epistemología del campo".

Durante este encuentro en la MSH, considerado actualmente como "reunión fundante" del campo, se creó un comité presidido por Jean Meyriat, compuesto además por 13 miembros, entre quienes se encontraban Robert Escarpit y Roland Barthes, al igual que C. Guillebau, Abraham Moles y F. Terrou. El Comité estableció como objetivo el reconocimiento de las CIC como disciplina universitaria y la creación de una sección al interior del Centro Nacional de la Investigación Científica (CNRS, por sus siglas en francés). Luego de varias gestiones infructuosas, el 28 de enero de 1975 se estableció un decreto que modificaba la composición del Consejo Consultivo Universitario (CCU), creando así la sección 52 ${ }^{a}$ Ciencias de la Información y la Comunicación (que en 1981 se convertiría en la sección $71^{\text {a }}$ ). Sin embargo, el elemento decisivo fue, durante el inicio escolar de 1975, la creación del primer DEA en la ahora Escuela de Altos Estudios en Ciencias Sociales. Otras escuelas se incorporaron en 1976, particularmente las universidades Bordeaux III, Grenoble III y París II. Los doctorados del tercer ciclo fueron habilitados al mismo tiempo; sin embargo, estas resoluciones no funcionaron de la misma manera para los doctorados de Estado y hubo que esperar la reforma de 1984 para que se diera origen al "nuevo doctorado", institucionalizando en el tercer ciclo a las CIC. En el CNRS, el objetivo no se alcanzó.

El Comité de los "padres fundadores" organizó en noviembre de 1975 el coloquio Relaciones entre las ciencias de la Infor- 
mación y la Comunicación, en cuyas actas, publicadas en 1977, se daba cuenta de las aproximaciones francesas de la investigación en CIC. En 1978, el Comité se convirtió en una sociedad sapiente y cambió de nombre por el de Sociedad Francesa de Ciencias de la Información y de la Comunicación (SFSIC), y organizó en la ciudad de Compiègne, ese mismo año, el primer congreso Inforcom. Posteriormente, la SFSIC organizó cada dos años un congreso que reunía a investigadores, profesores-investigadores, profesionales y doctorantes en cic, durante los cuales las contribuciones científicas y los intercambios daban cuenta del estado de la investigación en CIC y de la evolución de la disciplina. El XII Congreso sobre Emergencias y continuidad en las investigaciones en Información y Comunicación tuvo lugar en la sede misma de la unESCO, en París del 10 al 13 de enero de 2001.

En 1982, Armand Mattelart e Yves Stourdzé, por petición del ministro de Investigación e Industria, Jean-Pierre Chevènement, revisaron un estado de la cuestión sobre la investigación en CIC en Francia, remarcando una considerable alarma sobre el atraso acumulado en comparación con la situación de los países anglosajones. Estos autores señalaban que, a pesar de la importancia del desafío "económico, industrial, pero también y, sobre todo, social y cultural, la comunicación emergía a duras penas en Francia dentro del organigrama de las disciplinas científicas". Además, agregan, "esta ausencia de legitimidad contrasta con la pletórica presencia del tema de la comunicación y de las nuevas tecnologías en el debate político y en la vida cotidiana". Adelantaron una serie de propuestas para organizar institucionalmente la formación y la investigación en cIc, que no tuvieron ningún seguimiento.

Diez años más tarde, en marzo de 1993, el Comité Nacional de Evaluación (CNÉ) publicó una Relación de Evaluación de las Ciencias de la Información y de la Comunicación, dando cuenta de los progresos realizados, a pesar de la fragmentación de investigaciones en varias secciones y del rechazo del CNRs para "otorgar a los investigadores especializados en información-comunicación, el lugar y los medios específicos a los cuales ellos consideraban tener 
derecho". En ese mismo tiempo, la Dirección de la Investigación y Estudios Doctorales del Ministerio de la Educación Nacional (DRÉD, por sus siglas en francés) reconocía 24 equipos de investigación, "número bastante más limitado que el de la enseñanza universitaria de primer y segundo ciclo".

En ese mismo informe del CNÉ, se señala que la investigación pública independiente de las universidades era importante. La investigación se desarrollaba en grandes instituciones (principalmente en el Instituto Nacional de lo Audiovisual y en el Centro Nacional de Estudios de las Telecomunicaciones), o en algunas otras estructuras (Instituto de lo Audiovisual de Telecomunicaciones en Europa, con base en Montpellier, Asociación Descartes).

El rechazo del CNRS a incorporar las CIC continuó aun y cuando a finales de 1999 tres secciones del Comité Nacional declararon, con cierta timidez, incumbencia por la comunicación. Solamente el Laboratorio Comunicación y Política -que en 1998 festejó su décimo aniversario- contribuyó, a través de su revista Hermès (cuyo subtítulo es Cognición, comunicación y política) a incrementar la reflexión en sus campos de competencia. En enero de 2001 se creó un nuevo departamento: Ciencias de la Información y de la Comunicación Tecnológicas (sICT, por sus siglas en francés). Este departamento tiene una orientación eminentemente tecnológica, distanciándose, una vez más, de los enfoques de las ciencias sociales y humanas.

Desde la institucionalización de las CIC en 1975, se registró un crecimiento regular de profesores-investigadores reconocidos por la sección $71^{\mathrm{a}}$, constatándose a partir de ahí una evolución del campo disciplinar. De 27 profesores en el origen, el número se incrementó a 257 en 1992, a 363 en 1996 y a 450 en el año 2000 (a ese número habría que agregar un $10 \%$ de no titulares, encargados de curso y de investigación, y los profesores asociados de tiempo parcial que ejercen semi-servicios por periodos de tres años renovables). Una de las características de estos universitarios es que se encuentran, indistintamente, integrados a las facultades de Letras, de Ciencias Humanas, de Derecho e incluso de 
Ciencias Políticas. Esto es una evidente prueba del distanciamiento institucional de este campo de actividad.

En 1993, la 71 a sección del Consejo Nacional de Universidades ( $\mathrm{CNU}$ ) elaboró un documento en el que definía los "campos de competencia de las ciencias de la información y de la comunicación". El texto (modificado el 11 de junio de 1999), difundido posteriormente sin interrupción, constituye un marco de referencia y considera lo que las cIC abarcan:

1. Los estudios sobre las nociones de información y de comunicación, sobre sus relaciones, sobre la naturaleza de los fenómenos y de las prácticas que así se denominen, al igual que los diferentes enfoques científicos que apliquen.

2. El estudio, por una parte, del funcionamiento de los procesos, de las producciones y de los usos y, por otra, de la recepción, de la información y de la comunicación.

3. El estudio de los actores individuales e institucionales de la información y de la comunicación.

4. El estudio de la información, de su contenido, de sus propiedades, de sus efectos y de las representaciones que ella vehicula.

5. El estudio de los sistemas de información y el de los sistemas de acceso a la información, documentales o no, informatizados o no.

6. El estudio de los medios de comunicación bajo sus diversos aspectos.

El campo de las Cic ha sido considerado por la $71^{\text {a }}$ sección como "decididamente interdisciplinario". Sin embargo, esto ha generado debate al interior de las SFISC para determinar si las CIC son una disciplina, una interdisciplina, una encrucijada, o...

"No hay duda sobre el hecho de que la demanda social de parte del gran público en relación con los especialistas en Ciencias de la Información y de la Comunicación, no hará sino crecer en razón de los constantes progresos tecnológicos en materia de comunicación”, subraya Rémy Rieffel. El advenimiento de una sociedad de información demanda, de los poderes públicos, un nuevo enfoque institucional y una voluntad sobre la verdadera 
estrategia de investigación en el campo de las Cic. La decisión del Ministerio de la Investigación, este año, de crear la MSH de París Norte, fundada sobre la base de un enfoque científico establecido por Pierre Moeglin, avanza en esa dirección.

\section{LOS ORGANISMOS PÚBLICOS}

Janine Delatte, Anolga Rodionoff y Marie Thonon

Evaluar la investigación en ciencias de la información y la comunicación (CIC, aunque recientemente se les denomina con frecuencia infocom), no es una tarea sencilla.

Tal y como lo saben las cic desde su nacimiento, las barreras disciplinares no han terminado de desplazarse, de que los investigadores se reconviertan y de que las tecnologías mezclen las pistas. En efecto, el fenómeno social que modifica todas las identificaciones clásicamente consideradas, se observa también en el campo de la investigación de las Cic.

De esta manera, si pretendemos dar cuenta de las tendencias y carencias de la investigación, nos veremos obligados a desbordar la conceptualización de referencia y a reagrupar objetos que no están considerados ahí.

En Francia, la investigación en comunicación prácticamente está financiada en su totalidad por el sector público, y se apoya en dos instituciones mayores: el CNRS y la Universidad. De hecho, con frecuencia estas instituciones son muy independientes entre sí, aunque hay algunos momentos en que se encuentran imbricadas. Sin embargo, se puede constatar que cada vez son más frecuentes las imbricaciones.

Así pues, se trata de dos polos que hemos explorado, no obstante conocer el hecho de que hay otros grupos de investigadores que se encuentran en estructuras más o menos formalizadas (redes de investigadores, despachos de estudios privados, despachos de asesores privados) y que intervienen en el campo de la comunicación. De la misma forma, los ministerios y otros organismos públicos (Investigación, Municipalidad, Cultura, Telecomunica- 
ciones, Educación Nacional, Consejo Nacional de lo Audiovisual, etc.), en ocasiones solicitan informes o muestreos que tienen en principio, como objetivo, informar sobre las políticas y aclarar su gobernabilidad. En el cNRS y en la Universidad, es pertinente agregar a las grandes escuelas (Politécnico, Escuela Práctica de Altos Estudios en Ciencias Sociales, Escuela de Minas), al Conservatorio Nacional de Artes y Materias (CNAM), al Centro Nacional de Estudios en Telecomunicaciones (CNET, mismo que constituyó el relevo de France Telecom R\&D, que sigue una lógica de carácter comercial), al Instituto Nacional de lo Audiovisual (INA), estos dos últimos se encuentran esencialmente presentes por sus revistas Réseaux y Dossier de l'audiovisuel que, por cierto, constituyen una relación implícita entre numerosos investigadores.

En términos de equipos de investigación en CIC, la abundancia universitaria es un hecho impactante y contrasta con las rarezas en el CNRS. En efecto, un único laboratorio se puede observar con claridad bajo el denominativo Comunicación y Política (creado y dirigido por Dominique Wolton hasta el año 2000; el actual director es Georges Vigneaux) que cuenta con una presencia pública e internacional a través de su revista Hermès y, recientemente, por su colección en comunicación.

Sin embargo, muchos otros investigadores forman parte de las CIC, o bien, trabajan temas que las destacan. Se les puede observar en cualquier centro, algunas veces en relación con un eje de investigación que reúne a un equipo; en ocasiones también en relación con un tema conducido por un solo investigador.

En lo relativo a los equipos universitarios, en su mayoría están constituidos por investigadores especializados en cic. Cuando no es así, aun cuando el campo constituya el eje principal de sus investigaciones, se encuentran presentes en ciencias políticas, ciencias de la educación, ciencias sociales, artes plásticas, cine, literatura, geografía. Los ejes de investigación o problemáticas, se asocian en su gran mayoría con el gran bloque de Ciencias del Hombre y de la Sociedad (política, socioeconómica, sociotécnica, por ejemplo, o antropológica) y, en otros casos, con las Ciencias del Lenguaje (esencialmente semiótica). En ocasiones, con las ciencias 
cognitivas y, con cierta rareza, con la filosofía o el psicoanálisis. Sin duda alguna existen otras asociaciones; sin embargo, no aparecen con claridad, y en consecuencia, no se han establecido. Los análisis que se han realizado, han utilizado los conceptos y métodos, aun y cuando tanto los enfoques como la mirada establecida sobre los objetos de investigación tengan como objetivo esencial, dar cuenta de las nuevas relaciones creadas entre las sociedades, los individuos y los soportes y sistemas de comunicación.

No es nuestro objetivo presentar una cartografía exhaustiva de los equipos de investigación en cIc. Esta observación resulta muy laboriosa y, para ello, se requeriría la posibilidad de explorar de manera fina el terreno bajo las etiquetas o palabras clave anunciadas. Nos parece, igualmente, que la investigación debería ser abarcada bajo el ángulo de sus resultados y de sus producciones (enfoques, coloquios, revistas, publicaciones, audiovisuales, sites, etcétera). En efecto, nuestro enfoque nos ha permitido medir el hecho de que con frecuencia detrás de algunos investigadores de renombre, vegetan pequeños equipos de jóvenes investigadores que encuentran muchas dificultades para financiar sus trabajos. Habría también que incluir la permanente compilación que constituye la gris literatura de las tesis. Una buena cantidad de equipos o de investigadores se esfuerzan por tratar de encontrar prolongamientos editoriales o de algún otro tipo para sus investigaciones, lo que en ocasiones refuerza actitudes individualistas poco relacionadas con los proyectos de un equipo que con frecuencia, a causa del limitado financiamiento, no logran concluir.

Forman parte también de este punto de vista el volumen y la reaparición de los financiamientos en cuanto a la decisión de los objetos que genera como la investigación pública en CIC debería apreciarse. Una de las mayores carencias identificadas, a saber, la investigación internacional, encontraría sin duda ahí su razón.

No obstante, a pesar de este crecimiento de equipos, se puede constatar que con frecuencia se encuentran poco ligados entre sí, circunstancia que genera una disparidad de su notoriedad y de sus medios. Sin embargo, su amplio número de investigadores 
existe. De esta manera, hemos podido poner al día los sectores y los temas que constituyen las preocupaciones mayores de los investigadores, y producir así una cierta legibilidad del campo.

Grandes tendencias se presentan en este escenario y hacen posible su interpretación con el objeto de seguir nuestra preocupación, a saber, descubrir las carencias que estimamos importantes en este panorama, visto en su conjunto.

De esta forma, establecimos una clasificación en función de los sectores abordados, circunstancia que permite reagrupar los temas enunciados por cada equipo o laboratorio, así como las problemáticas consideradas por los investigadores. Bajo este ángulo, dichos criterios permiten obtener con mayor fineza los objetos que ellos han manifestado como de mayor interés.

Dos sectores se destacan de entre todos: el de los medios tradicionales y el de los "nuevos medios" que, de hecho, reagrupan al conjunto de las nuevas tecnologías de la comunicación y de la información. Los otros sectores en grado de interés son la cultura y la comunicación, la educación, la comunicación empresarial y de las organizaciones públicas y, luego, los territorios y el arte. Esta categorización no impide los cruzamientos entre sectores diferentes, la emergencia de nuevas tecnologías de la comunicación y de la información, que para nada son extrañas, lejos de eso, a las interrogantes de los investigadores en materia de educación, por ejemplo, en particular sobre la difusión de saberes. De igual forma, el sector de los medios tradicionales se intersecta con el de las organizaciones públicas y privadas.

\section{Los medios tradicionales (audiovisual, prensa y radio)}

Entre los medios estudiados, el audiovisual (TV nacionales, privadas, así como las públicas en gran parte) parece ser el tema que domina, en tanto que la radio o el teléfono se encuentran poco presentes. Una gran parte de investigadores privilegian el estudio de los medios en Francia y algunos otros lo hacen dentro de una perspectiva europea, mientras que otros exploran los medios africanos, asiáticos (chinos), árabes, rusos y otros. 
1. Espacio público y comunicación. En este rubro se trabaja el tema de la democracia y de la opinión en su relación con los medios así como el tema de los efectos de los medios en los comportamientos políticos y sociales.

2. Economía del sector. El binomio regulación/desregulación o reglamentación/desreglamentación, que cuenta notoriamente con los actores públicos y privados que constituyen la internacionalización de la comunicación.

3. Recepción y público. No abusemos del término. Estos temas que han sido muy desarrollados por los investigadores anglosajones, se han integrado recientemente en Francia (como prueba tenemos la revista Hermès, que publica a numerosos investigadores extranjeros anglosajones para dar respuesta al tema de la recepción). Si pretendemos, en el sentido ordinario, en Francia se trata esencialmente de estudios de audiencia y de investigaciones sobre la noción de público (en especial en relación con la televisión).

4. Análisis del discurso. En el enfoque sociosemiótico trabaja el discurso de los medios (prensa y televisión esencialmente) y las representaciones centradas en lo audiovisual.

5. Sociología de los contenidos. De la producción audiovisual pública y privada, por una parte, y del tercer sector, por otra. Sin duda se trata de una particularidad francesa que se apoya mucho en el análisis de las representaciones realizadas por encuestas y entrevistas.

6. Historia de los medios. Clásico en ciertos sectores (el libro), se ha desarrollado mucho en el conjunto de los medios en la sociedad francesa, marcando la conjunción entre estructuras de comunicación y estructuras sociopolíticas.

7. Usos. Apoyándose en un saber sociológico importante en relación con el análisis de las prácticas como moradoras de usos, la presión y las afluencias de las nuevas tecnologías que ponen en ruta la investigación de campo y los temas de apropiación de las técnicas en los usos.

8. Profesión. Un sector largamente estudiado: la prensa, en particular los periodistas. 
Estos diferentes temas de investigación combinan los cuatro ejes (económico, histórico, relación con la técnica y los objetos y significaciones) que observaba Thierry Lancien a partir de un corpus de tres textos. Sin embargo, subrayemos que si los ejes económico e histórico (temas 2 y 6) se observan sin dificultad, los otros dos, el de la relación con la técnica y los objetos, y el de las significaciones, se cruzan con frecuencia en tanto que uno llama al otro y, aún más, no se separan. Los demás temas repertoriados participan en estos dos últimos. Por último, el sector de los medios tradicionales es abordado tanto por los equipos como por laboratorios de la Universidad y del cNRS.

\section{Los nuevos medios}

La noción de los "nuevos medios" reenvía a la de nuevas tecnologías de la comunicación y de la información (NTCI), que es el término más frecuentemente utilizado, o algunas veces multimedia o de forma menos usual, a la de Internet. Hay dos temas que dominan con amplitud a los otros. Se trata de los usos y de las técnicas. Contrariamente a los medios más antiguos, el eje temático está muy presente. Aun y cuando observemos un interés particular por la gestión, el análisis de las innovaciones tecnológicas y sus incidencias tanto en el dominio económico como en el social sigue siendo el enfoque privilegiado. Este sector de los nuevos medios con frecuencia cruza otros como el de la educación, el arte y el territorio.

1. Usos. En este caso, el término mismo se entiende en un sentido amplio si consideramos tanto la vertiente de las innovaciones como la de la prospectiva. De manera intencional reunimos los dos tipos de usos: por una parte, aquellas que resultan de las innovaciones relacionadas con la apropiación social de las NTCI y por otra aquellos que, finalmente, se dan conforme a la funcionalidad prevista por los ingenieros y los técnicos.

2. Técnicos. Con frecuencia tratan sobre el cuestionamiento de los flujos de informaciones o de redes o reflexiones sobre su gestión. Si la noción de red es polisémica, generalmente nos 
reenvía, en este caso, a su versión técnica. Ésta es una de las razones por la que ordenamos todas las cuestiones relativas a la red en el eje técnico. De igual forma algunas otras perspectivas se han han podido considerar como el "tratamiento automático de las lenguas naturales" o la "modelización de la comunicación hombre/máquina” y, también, la “ergonomía de las NTCI" y algunas más.

3. Políticas públicas a escala nacional o local.

4. Historia de las ciencias y de las técnicas de las máquinas de comunicar.

5. Derecho y nuevas tecnologías de comunicación y de información. Se analiza la evolución del sistema jurídico frente al desarrollo de las NTCI como asuntos relativos a la propiedad intelectual.

6. Economía y tecnologías de la comunicación y de la información. Reorganización del sistema productivo frente al cambio tecnológico y nueva configuración de oficios y profesiones.

7. Recepción.

Los usos y las técnicas muestran el eje de relaciones a la técnica y a los objetos, así como el eje de las significaciones. Políticas públicas, derecho y economía de las NTCI entran en el eje económico y finalmente, historia de las ciencias en el eje histórico.

El análisis de los contenidos parece estar prácticamente ausente salvo en algunas excepciones: el estudio de las imágenes de síntesis, el de la literatura con la producción de textos electrónicos, o el de las actividades artísticas. Clasificamos a la primera en el sector cultura y comunicación bajo la temática imagen, y los dos siguientes en el sector artístico. El sector de los nuevos medios, según parece, suscita más el interés de los investigadores en la Universidad y en las grandes escuelas.

\section{Cultura y comunicación}

Varios cruces de interés se efectúan entre estos sectores. Subrayamos algunos de estos temas: 
1. Modernización técnica y cambios culturales.

2. Industrias culturales.

3. Representaciones.

4. Multiculturalidad.

5. Cine documental.

6. Imagen (mecanismo de decodificación de la imagen fija y animada por ejemplo, o estética de las imágenes).

7. Identidad.

Los temas 1, 5 y 6 remarcan el eje de relación con la técnica y los objetos y el eje de las significaciones. El tema 2, el eje económico, aunque también el histórico. Finalmente, los temas 3 y 6 el eje de las significaciones, que constituyen también un sector presente en la Universidad, en el CNRS y en las grandes escuelas.

\section{Educación}

1. Mutación de los conocimientos como consecuencia de la evolución técnica. En este tema se pueden encontrar asuntos relativos a los usos y a la recepción.

2. Redes de difusión de los conocimientos dirigidos al gran público así como es desarrollado entre investigadores, en términos técnicos (bibliometría y otros).

3. Mediación de conocimientos especializados en relación con los contenidos.

4. Profesiones, esencialmente relacionadas con la documentación o la enseñanza.

Estos cuatro temas se agregan al eje de relación con la técnica y con los objetos y al eje de las significaciones. Es un sector en el que los universitarios muestran un interés mayor.

\section{Comunicación empresarial y organizacional}

1. Publicidad.

2. Mercadotecnia. 
3. Reorganización y nuevos modos de gestión frente a las estrategias de comunicación empresarial o de colectividades locales.

Estos tres temas se agregan al eje económico. Es un sector que también está presente tanto en la Universidad como en el cNRS así como en las grandes escuelas.

\section{Territorio}

1. Fomento de los recursos territoriales. Dinámica urbana y la recomposición en territorio bajo el efecto de las NTCI.

2. Políticas públicas.

3. La ciudad, frecuentemente asociada a las políticas de relación social.

Los temas 1 y 3 subrayan el eje de relación con la técnica y con los objetos, en tanto que el tema 2 el eje económico. Este sector se encuentra presente tanto en la Universidad como en el CNRS.

\section{Arte e hipertexto}

1. Estética de la imagen digital.

2. Prácticas artísticas en la Internet.

3. Historia de las imágenes. Relaciones imágenes digitales/imágenes plásticas.

4. Museología.

5. Música (IRCAM).

6. Historia social de la música.

7. Literatura e interactividad.

Todos los temas destacan el eje de relación con la técnica y los objetos y con el eje de las significaciones a excepción de los temas 3 y 6 , que se ubican en el eje histórico. Se trata aquí de un sector muy desigual en su planteamiento en razón de que sólo los universitarios se interesan en él. La música hay que considerarla aparte. 
Este breve panorama, que incluye centros de investigación y laboratorios del CNRS y las grandes escuelas, así como equipos y laboratorios de investigación universitarias ligadas a los DEA, da cuenta de hasta qué punto de dispersión se encuentra actualmente la investigación en información y comunicación. Así, no podemos hablar de tendencias fuertes bajo el ángulo de una voluntad política de investigación. Por lo que respecta al CNRS, las direcciones actuales de investigación parecen estructurarse entre categorías antiguas y proyectos más o menos individuales. Estas tendencias de investigación no parecen ser sino la forma de reagrupaciones o de competencias sobre los temas insignia en el interior de las instituciones, sin que correspondan necesariamente a las necesidades o a las urgencias de investigación de la sociedad francesa, frente a los desafíos del cambio. Desde julio de 2000, el cNRs formuló de manera oficial la creación de un campo de información y comunicación, centrado en las nuevas tecnologías. Sin embargo, ¿en qué parte estarían presentes dentro del campo de las ciencias sociales?

En lo referente a las investigaciones universitarias, la heterogeneidad de los temas abordados por ciertos equipos o laboratorios, indican claramente que las decisiones acerca de la dirección o sectores a explorar no constituyen sino decisiones particulares. Sin embargo, se alcanza a despejar una cierta coherencia más clara por medio de los temas que plantean los nuevos equipos. Esta característica se debe, muy probablemente, a la naturaleza más cualitativa de las informaciones recibidas.

Parece que dos sectores se destacan con más claridad que los otros: el de los medios tradicionales y el de los nuevos medios. Este último se entrecruza con otros sectores como la literatura, el arte, el territorio, la educación o la comunicación en el interior de las empresas privadas como organizaciones públicas y que se consideran de acuerdo con las perspectivas técnicas, simbólicas o económicas. Estas cuatro corrientes de investigación se destacan entre los temas abordados, aunque de manera muy desigual. Así, el eje relación con la técnica y los objetos, y el de las significacio- 
nes, dominan ampliamente y se encuentran presentes en prácticamente todos los sectores. En seguida, encontramos el eje económico, que parece estar cada vez más presente en las estructuras de investigación ligadas a las grandes escuelas. Finalmente, el eje histórico parece ser el menos tratado.

A pesar de los diferentes enfoques, algunos se encuentran presentes muy tangencialmente. Es el caso, por ejemplo, de los enfoques de carácter psicoanalítico y filosófico.

Así, si consideramos el ángulo de las carencias, se pueden observar diferentes aspectos que nos parecen fatalmente ausentes y de los que algunos ciertos jóvenes investigadores se han dado cuenta (La lettre d'Inforcom núm. 22, 2000. Texto de P. Lardellier).

- Desigualdades sociales frente a las nuevas tecnologías.

- Estudios europeos comparativos. Internacional.

- Sociedad de la información: transparencia, secreto y seguridad.

- Producción audiovisual: oferta pública nacional y europea, y análisis de la oferta.

- Mujeres, minorías y medios.

- Movimientos sociales y medios (Internet).

- Arquitectura.

Sin ninguna duda la lista debe estar incompleta; sin embargo, sólo un programa refundador podría trabajar la reconsideración de la medida de los cambios actuales y establecer, por medio de la investigación, los instrumentos necesarios para repensarlos. Por último, podemos subrayar que este panorama no es exhaustivo en la medida en que las informaciones de las que disponemos aún contienen algunas lagunas. En consecuencia, nuestras conclusiones son relativas e indican, de manera general, las grandes corrientes y sectores de la investigación.

Sin embargo, consideramos que es suficiente para evidenciar el hecho de que no se ha establecido una verdadera política de investigación en el seno de los poderes públicos. Se puede apreciar que el desarrollo actual de la investigación que reconoce a la 
comunicación como paradigma de la recomposición social, muestra que hay una movilidad temática real afirmada por el CNRs; de igual forma, se observa que las direcciones actuales no parecen aún constituir el hecho de reagrupamientos institucionales más o menos coherentes o de proyectos más o menos incluyentes.

Créditos y estudios con frecuencia son disparates y parecen corresponder poco a la función de expertise frente al Estado, en el que la investigación también podría asegurar. Tal y como lo afirma el señor Dagnaud, a propósito de los medios audiovisuales, podemos concluir que

Existe un malentendido en cuanto a las relaciones entre el Estado decisor y la investigación universitaria: teóricamente éste dispone como herramientas de experiencia para la acción, de los conocimientos que emanan de la Universidad y del cNRs; en la realidad, en todos esos casos este dominio, estos dos mundos se ignoran casi en su totalidad.

Esto, sin duda, permite comprender la dispersión de los estudios y el poco impacto que pueden tener sobre las instancias de decisión, así como su distanciamiento con la demanda social y la realidad de los cambios a conducir.

\section{LAS REVISTAS}

Thierry Lancien

Al escoger las revistas consagradas a la comunicación con el objeto de encontrar los indicadores de lo que pudieran ser las grandes corrientes de la investigación del campo no intentamos, sin embargo, realizar una búsqueda exhaustiva en las diferentes revistas. En términos de método, requeríamos establecer criterios para delimitar un territorio a repertoriar, circunstancia que realizamos estableciendo una mirada a las grandes corrientes de investigación en los términos en que son evocadas en tres textos consagrados a este tema, con el examen de cuatro revistas del campo que escogimos con los criterios que hemos señalado. 
En lo referente a los textos partimos, para obtener una cierta perspectiva del Reporte de evaluación de marzo de 1993, y luego, cuando confrontamos en dos entrevistas a investigadores en 1999. Estos elementos deberían permitirnos observar cuáles eran los grandes temas que se despejaban sobre el campo, pero también si hubiera, en esos seis años, temas que permanecían o cambios importantes.

Para la elección de revistas, decidimos retener aquellas que eran citadas con mayor frecuencia en cuatro textos o documentos, contando aun, para la puesta en perspectiva, un documento de 1992, el Cinémaction. Los otros tres textos fueron el libro de Dominique Wolton, Pensar la comunicación, el artículo de Cécile Méadel, consagrado a las revistas y publicado en un número de Dossier de l'audiovisuel especializado en la investigación en comunicación y, finalmente, los elementos bibliográficos de este mismo número. Este minicorpus fue heterogéneo a propósito; sin embargo, lo consideramos lo suficientemente representativo pues permite hacer cruzamientos de las informaciones surgidas de escritos con estatus de carácter diverso. De esta forma, encontramos que las revistas de mayor peso en el campo de las referencias eran cuatro: Les dossiers de l'audiovisuel, Hermès, Quaderni y Réseaux.

Es necesario precisar que con este método no nos propusimos establecer criterios como los relativos al carácter científico de una revista. Sabemos, por otra parte, que este asunto es complejo y remitimos desde esta perspectiva a los trabajos de Robert Boure y, más generalmente, para las revistas del campo, a los trabajos desarrollados en el marco del grupo TPS de las SFSIC.

\section{Los grandes ejes de la investigación en los tres textos}

El examen de los tres textos muestra, para comenzar, una permanencia de los temas de investigación de 1993 hasta la actualidad (dos campos continúan siendo los mismos) y en seguida, grandes proximidades en los ejes avanzados en los dos textos de 1999 (dos campos continúan siendo parecidos). 
En el Reporte del comité nacional de evaluación, los tres grandes ejes evocados son los siguientes:

1. Relación con la técnica y los objetos. Análisis de las máquinas de comunicar. Sociología de la innovación. Examen de la apropiación de las técnicas y estudios de usos.

2. Temas de significación. Estudio de las representaciones construidas en y por los medios. Recursos de la pragmática y de los estudios lingüísticos de las interacciones. Producción del sentido por los medios. Construcción de la opinión.

3. Economía del sector. Políticas públicas y privadas. Internacionalización de las industrias culturales. Reglamentaciones.

En la entrevista a Bernard Miège en los Dossier de l'audiovisuel, él establece dos grandes ejes:

1. Producción de mensajes. Producción de sentido y recepción de los discursos. Así pues, muy cercano al tema 2 que precede.

2. La mediación técnica. Innovación técnica. Producción de usos. Cercano al tema 1 precedente. A éstos, el autor agrega tres campos que, desde su perspectiva, deben explorarse:

- Reflexión sobre la sociedad de la información.

- Información especializada, científica y técnica.

- Intercambios mundiales.

Por otra parte, Bernard Miège agrega un cuarto eje: el estudio de las escrituras multimedias, que podríamos considerarlo en el tema 1.

Por su parte, Armand Mattelart menciona en la entrevista de Dossier de l'Audiovisuel, cuatro grandes ejes:

1. Economía política de la comunicación. Industrias culturales. Se podría incluir en el tema 3 del Reporte.

2. Usos sociales de las máquinas de comunicar. Mismo que podría incluirse en el tema 1 del Reporte y en el 2 de Miège. 
3. Análisis del discurso. Que se puede incluir en el tema 2 del Reporte y en el tema 1 de Miège.

4. Regreso a la historia. Por ejemplo, origen de la ideología de las telecomunicaciones o, aún más, la trayectoria y origen de las teorías.

Los temas de las revistas en relación con los grandes ejes de investigación

De entrada se puede constatar que, de 78 números de revistas, los dos grandes ejes comunes en los tres textos ("temas de significación" y "relación con la técnica") se encuentran bien representados en 33 números de revistas.

Dada la importancia de estos dos ejes, sería necesario afinar el análisis detallando los temas retenidos y desde luego, su presencia en las revistas.

El eje Economía del sector, presente en dos textos, se encuentra bien representado en once números de las revistas.

Por otra parte, los ejes evocados en un solo texto también pueden encontrarse en las revistas. Es el caso del eje Regreso a la historia (Mattelart), en cuatro números de revistas, y el eje Cuestionar la sociedad de la información (Miège), en un número.

Finalmente, en 26 números se abordan temas que no estaban presentes como parte directa de los grandes ejes. Sobre este aspecto, se deberían cruzar los datos con el tipo de revista puesto que, por ejemplo, aparecen temas temas relacionados con la materia, sobre todo en la revista Quaderni (25 números).

Al margen de la consideración de las características de las revistas, sería necesario orientar la atención hacia las fechas de aparición para ver si existen en los años "picos de interés" para algunos de los temas.

Gracias a la apreciación operada entre los ejes y revistas, se puede observar con claridad que: 


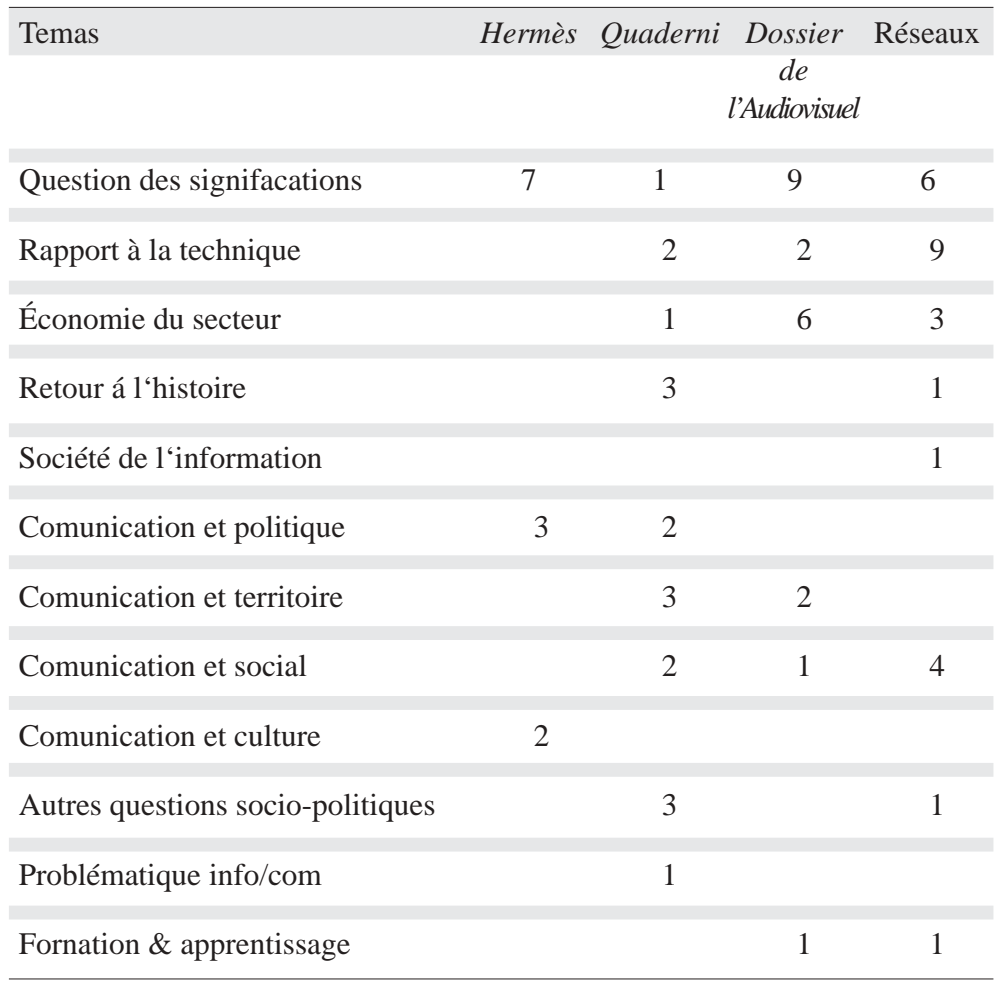

- Los principales ejes evocados en los textos se encuentran, en efecto, ampliamente representados en los seis años en las revistas analizadas.

- Se encuentran mayoritariamente presentes en dos revistas (Les Dossier de l'audiovisuel y Réseaux), en menor proporción en otras (Hermès) y, muy escasamente, en una última (Quaderni).

\section{UN CONCEPTO NUEVO: LA INTERMEDIALIDAD}

Gilles Delavaud

Entre los temas abordados con mayor frecuencia durante estos últimos años en las revistas no especializadas en comunicación, 
se puede encontrar una interrogante que insiste en la noción de la imagen (considerada como un punto de vista filosófico, jurídico o teológico), en la noción de dispositivo (en particular en el campo de las prácticas artísticas) y, más recientemente, en la noción de intermedialidad. Esta última da lugar a diferentes corrientes de investigación entre las cuales las más vivas son aquellas que privilegian ya sea la genealogía de los medios o bien, más ampliamente, la relaciones entre los medios.

\section{Genealogía de los medios}

El discurso sobre la convergencia de los medios no se realiza, sin duda, para nada en el caso actual del término intermedialidad. Es necesario, sin embargo, no confundir el segundo término con el primero. Forjado por Jürgen E. Müller en 1987, el concepto de intermedialidad se refiere a una problemática específica: la de la identidad de los medios y, en consecuencia, de su desarrollo histórico con lo que ello supone de interferencias recíprocas. Müller establecía, de esta manera, la hipótesis de que "la televisión debe ser concebida como un dispositivo híbrido e intermediático que se encuentra en las interacciones complejas con otros dispositivos". Al abordarse los medios en términos de identidad más que de especificidad, el enfoque los considera como procesos. Es en esta perspectiva como se sitúan las investigaciones de William Uricchio sobre la prehistoria y la historia de la televisión desde el último cuarto del siglo xIX. Ahí él plantea, por ejemplo, las siguientes preguntas: ¿cuál era el horizonte de las expectativas del público de finales del siglo XIX? ¿Cuál es el lugar de la televisión en relación con la radio, el cine o el teléfono? ¿Cómo explicar que el desarrollo del cine haya precedido al de la televisión? ¿En qué parte de la problemática del surgimiento de la televisión se sitúa el tema que aclare la aparición de los nuevos medios actuales?

El mismo autor, en varios estudios sobre el nacimiento de la televisión alemana en los años treinta, evidencia diferentes concepciones encontradas dependiendo del hecho de que a la televi- 
sión se le considere más bien como un perfeccionamiento del teléfono, de la radio o del cine: ¿el nuevo medio sustituía la audición o la visión? ¿La recepción debería ser individual o colectiva? ¿Qué papel se le podría hacer desempeñar a la televisión en la constitución de un nuevo espacio público al alba de la Segunda Guerra Mundial? De forma general, ¿el desarrollo de este medio no está ligado más al imaginario cultural que a las complicaciones tecnológicas?

El concepto de intermedialidad ha sido retomado actualmente por los historiadores del cine, ansiosos de proponer un modelo que pueda dar cuenta del hecho de que "la identidad del cine como nuevo medio fue de larga constitución". Siguiendo la definición de Rick Altman, la intermedialidad designa "una etapa histórica, un estado transitorio en el curso del cual una forma en vías de convertirse en un medio a parte se encuentra todavía dividido entre varios medios existentes, a un punto tal que su propia identidad queda en suspenso". Es bajo este espíritu que, utilizando al cine como "prototipo", como André Gaudreault y Philippe Marion construyeron su modelo del "doble nacimiento de los medios": un medio "nace una primera vez como prolongamiento de prácticas anteriores a su aparición y a los cuales estaba integrada en su etapa primaria [...] Y así, nace una segunda vez en cuanto toma una vía al seno de la cual los medios que ha desarrollado han adquirido esta legitimidad institucional que reconoce su especificidad". Esta hipótesis conduce a Gaudreault y Marion a sustituir el término ambivalente de nacimiento con otros tres términos de igual forma a designar los tres tiempos de gestación y de desarrollo de un medio: aparición (de una tecnología), emergencia (de un dispositivo), advenimiento (de una institución).

Por su parte, al estudiar el advenimiento del sonido en la industria cinematográfica en Estados Unidos, R. Altman muestra que para que un nuevo medio se "libere" del estadio intermediático inicial, se requieren tres operaciones sucesivas: la citación, la explotación y la separación. El nuevo medio -que al inicio no es sino una nueva tecnología- comienza por citar a los otros me- 
dios, no sólo para hacerse aceptar sino para mostrar, por medio de sus imitaciones, que puede desarrollar capacidades análogas a los medios ya existentes. Es necesario, en seguida, con el objeto de afirmar sus propias posibilidades, explotar a los medios antiguos y probar que es "capaz de realizar proezas que rebasan las capacidades de los otros medios". Finalmente, para establecer su especificidad y estabilizar su identidad, requiere desmarcarse de los otros medios, separarse, mostrándose en todo caso capaz, a partir de su formación, de integrar a los otros medios a su propio marco. Sin embargo, la estabilidad y la legitimidad institucional de un medio no se obtiene de manera definitiva por siempre en virtud de que, como lo señala Altman, "una nueva intermedialidad está siempre latente". Actualmente podemos observarlo con la televisión, cuya identidad se encuentra "comprometida por la intermedialidad informativa".

Desde 1999, el Centro de Investigaciones sobre la Intermedialidad de la Universidad de Montreal, organiza cada año un coloquio internacional dedicado a La nueva esfera intermediática. El segundo coloquio co-organizado el año 2000 con el Observatorio del recuento mediático, de la Universidad Católica de Louvain, tuvo como tema "El recuento en la época de la cultura mediática: mutaciones y rupturas".

\section{Relaciones entre medios}

A partir de otro tipo de investigaciones, la revista Degrés organizó un número reciente titulado "Intermediality". En este caso, el término designó "interdisciplinariedad entre las artes" - especialmente sobre las artes del espectáculo- en su carácter de medios. El artículo de Philip Auslander: "Liveness, Mediatization and Intermedial Performance" es un artículo representativo de esta corriente. Luego de analizar los intentos principales de integración de la imagen filmada en el espectáculo en vivo, desde el inicio del siglo Xx, el autor cuestiona, por una parte, el papel determinante desempeñado por los medios, en particular de la tele- 
visión, en la economía del aporte de la música y, por otra, el sentido y la inducción sistemática del uso de las pantallas gigantes de video en los eventos deportivos o en los conciertos, y la transformación de las condiciones de la experiencia que sobre estos aspectos resultan en el espectáculo. El autor muestra que la televisión ya no es únicamente "una dimensión de nuestro ambiente, un discurso entre otros sino que debe ser considerada como una ambiente en sî" que ha "trascendido su identidad como medio específico que ha invadido la cultura"; lo televisual forma a partir de entonces "parte integrante de la fábrica social". En ese contexto en donde la "presencia" de hecho se encuentra devaluada, la televisión ya no se contenta con transmitir formas culturales anteriores, sino que la televisión "las convierte en televisión".

\section{LAS LISTAS DE DISCUSIÓN EN INTERNET}

\section{Hélène Cardy y Pascal Froissart}

El estudio de los temas que privilegian los jóvenes investigadores, o sus temas de preocupación, no es algo fácil. La existencia en Francia de una "lista de discusión" en Internet que se les ha dedicado representa, pues, una interesante puerta de entrada. Observando los intercambios de jóvenes investigadores entre ellos, se puede conocer a los que serán los futuros miembros de las ciencias de la información y la comunicación, al margen de cualquier jerarquía natural heredada de las estructuras académicas.

La lista de discusión que genera la reunión de los doctorantes y jóvenes investigadores se llama SICliste, que constituye un amable juego de palabras que se funda en las siglas sic (Ciencias de la Información y la Comunicación), que sin duda hacen referencia al trayecto combativo que representa la inserción del joven investigador en las estructuras universitarias. La lista está disponible exclusivamente en Internet, a través del correo electrónico.

La SICliste manifiesta claramente su apego a las problemáticas de la información y la comunicación. Está dedicada a los "estudiantes investigadores dedicados a las ciencias de la información y la comunicación". La lista tiene como objetivo la 
Difusión de mensajes cuyos contenidos están centrados en temas propios de los doctorantes en Ciencias de la Información y la Comunicación. Estos temas abordan aspectos prácticos del doctorado en SIC (informaciones relativas a los derechos, medios de los doctorantes, sus adscripciones, sus inserciones, la publicación de sus trabajos, etc.), así como los debates teóricos que nutren su práctica. Esta lista es un espacio de expresión, de cooperación y de convivencia.

Creada en 1995 por un doctorante de la Escuela Nacional Superior de Ciencias de la Información y de Bibliotecas (ÉNSSIB), Vincent Mabillot, la SICliste ha contado con poco menos de un centenar de miembros por año. Éstos, en su mayoría, son doctorantes en Ciencias de la Información y la Comunicación, tal y como lo evidencian los retratos publicados en línea como una forma de ritual de presentación (puesto en línea por el webmestre). La mayoría cuenta con alguna actividad de enseñanza (encargado de curso en París XIII, encargado de enseñanza [ATER] en el IFP, profesor en París XIII, etcétera).

En cinco años se intercambiaron alrededor de 1,200 correos electrónicos, según un periodo bastante aleatorio (que depende demasiado de la repartición de los turnos de diálogo, $c f$. Figura 1) a razón de 20 correos por mes en promedio.

El volumen total de correos electrónicos, aparentemente débil, no debe ocultar la gran disparidad de las frecuencias de intervención de unos y de otros. Como en toda dinámica de grupo, los intercambios son asimétricos ( $c f$. Tabla 1 y Fig. 2).

Algunos de los participantes en las listas intervienen de manera importante, otros lo hacen con menor proporción, llegando incluso a ninguna participación; sin embargo, no se renuncia al hecho de pertenecer a la lista.

En la SICliste, como en cualquier grupo organizado, el diálogo se organiza de manera equilibrada dentro del desequilibrio: una persona ocupa, ella sola, cerca de un cuarto de las comunicaciones; tres participantes realizan la mitad de las intervenciones. Se requieren sólo nueve para llenar los tres cuartos de las discusiones. Finalmente, un gran tercio (32 miembros) no participaron 


\section{FIGURE 1}

Volume MENSUEl DES MESSAGES (PAGES NORMALISÉES)

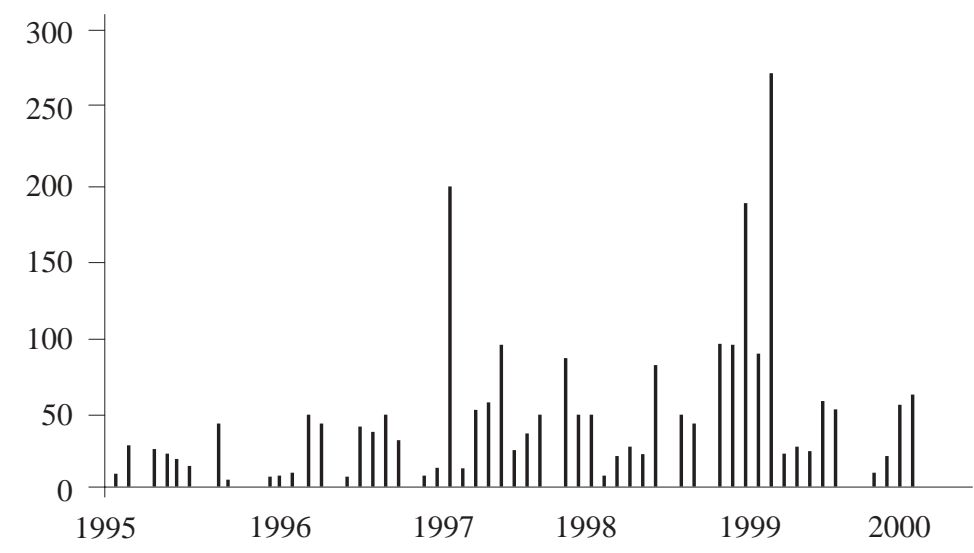

Fuente: Archives de la Sicliste. Traitement. H. Cardy \& P. Froissart, Université de parís VIII @ 2001.

FIGURE 2

TOURS DE PRISE DE PAROLE

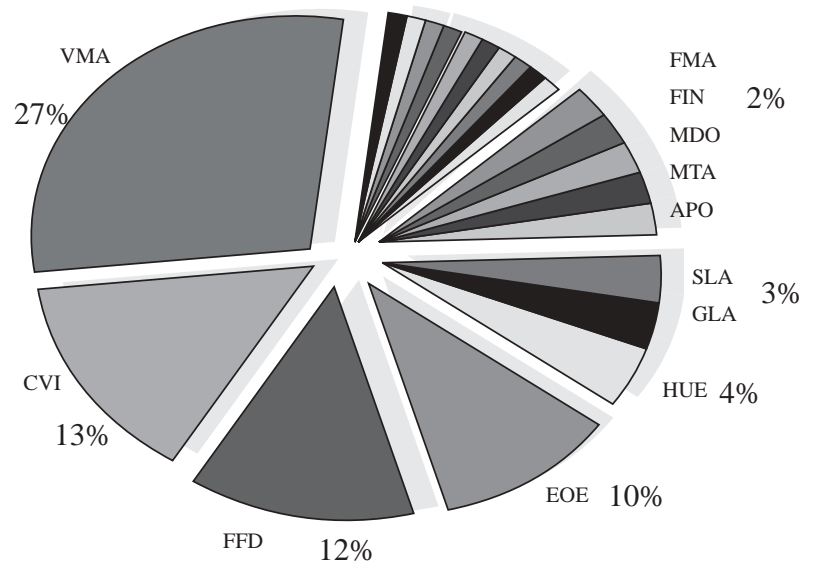

Fuente: Archives de la Sicliste. Traitement. H. Cardy \& P. Froissart, Université de parís VIII @ 2001. 


\begin{tabular}{lcc}
\hline \multicolumn{3}{c}{ TABLA 1 } \\
Tours DE PAROLES \\
Nombre de participants & \multicolumn{2}{c}{ Part. des interventions \% } \\
1 & 27 & $1 / 4$ \\
2 & 40 & \\
3 & 52 & $1 / 2$ \\
4 & 62 & \\
5 & 66 & $2 / 3$ \\
6 & 68 & \\
7 & 71 & \\
8 & 73 & $3 / 4$ \\
9 & 75 & \\
10 & 77 & \\
54 & 100 & \\
86 membres inscrits & & \\
\hline
\end{tabular}

Fuente: Archives de la Sicliste. Traitement. H. Cardy \& P. Froissart, Université de parís VIII @ 2001.

para nada en el intercambio. Silenciosos, éstos forman parte de lo que en la jerga informática se conoce como lurkers, quienes legitiman las listas por su número y dejan perplejos a los webmestres. Es claro que, como en todas las listas de discusión, la SICliste imita la dinámica de los grupos reales. La lista se estructura rápidamente en función de factores clásicos como la proximidad (real, en virtud de que los miembros de la SICliste no sólo se comunican por los medios virtuales si consideramos sus relaciones particulares, que se renuevan entre los participantes), la homogeneidad de los miembros tanto desde la perspectiva intelectual como afectiva, etcétera.

La originalidad en la SICliste se concentra, evidentemente, en observar los intercambios que ahí se desarrollan. Considerando que los archivos se encuentran abiertos, es posible intentar una radiografía rápida de los temas abordados a lo largo de las discusiones. De ahí resulta una temática dominante así como una serie de temas y de preguntas.

La constatación que se revela desde el inicio, reposa en el hecho de que los actores de la SICliste se interesan muy poco en 
los problemas epistemológicos que sacuden a las sIC: ¿qué es la comunicación?, ¿en qué consiste la pretensión de formarse en comunicación?, ¿cuál es la identidad de las SIC?, y otras. Estos temas no parecen de actualidad en la SICliste pero sin embargo, producen mucha efervescencia en el Landerneau de la investigación en comunicación (como los congresos y los grupos de estudio de la Sociedad Francesa de las Ciencias de la Información y la Comunicación, por ejemplo); sin embargo, en la lista no se tiene movilidad. ¿Significa esto que los jóvenes investigadores no se interesan por estos temas? Es posible, pero es mejor intentar ver en ello un efecto de generación: la mayoría de los miembros de la lista han realizado lo esencial de su formación desde el primer año universitario hasta el doctorado en cursos, o terminado en Ciencias de la Información y la Comunicación. Los jóvenes investigadores, formados desde el inicio en una interdisciplina, han podido así interiorizar la dificultad de definición hasta el punto de no imaginar a las ciencias de la comunicación menos sólidas sobre sus bases ontológicas que la sociología o la sicología o, al punto de dar poca importancia a este tipo de debates.

El mail de G. tiene dos méritos: nos informa sobre lo que ha pasado en el cierre del Coloquio de la SFSIC. En este correo se hace un llamado sobre un tema que transpiró a lo largo del coloquio: ¿qué cosa son las SIC? Me parece que, por una parte, el problema se encuentra en las palabras-valija que definen nuestro campo científico. Información y comunicación son, primeramente, víctimas de una apropiación abusiva por los medios de masa. Por otra parte, creo que es nuestro papel, de jóvenes investigadores en sIC, definir este campo. Somos la primera generación en ser legitimada en nuestro carácter de investigadores en SIC. Nuestros predecesores son los fundadores pero que provienen de campos científicos diversos (VMA, 01/12/96).

¿Por qué seríamos nosotros más legitimados que los otros? ¿Solamente porque hemos tenido un trayecto exclusivo en SIC? En efecto, es en todo caso una experiencia nueva. Y sobre este escenario habría que obtener algunas conclusiones, particularmente sobre el plano metodológico. Por el contrario, yo diría que en nuestro ca- 
rácter de jóvenes investigadores en SIC, debemos ser escuchados y eso es menos claro en este momento, es la impresión que tengo... y eso podría ser (cuestión de suerte) porque nos hemos empapado en esta famosa marmita de las sic. En ese caso, ¿podríamos ser deslegitimados por los "viejos" (GVI, 05/12/96).

No obstante, ¡somos afortunados de que las sic existan! Así, se trata de una disciplina sobre la que se puede hacer una tesis que puede ser presentada tanto en el campo de la psicología, sociología o aun en ciencias políticas, aunque en el caso en el que puede prevalecer un perfil, más bien tortuoso, es en las ciencias de la comunicación. En mi caso preciso, tendría dificultades para contestar las preguntas que me planteé si hubiera enfocado la "máquina" un poco más de cerca. De igual forma, hubiera tenido dificultades para entender el lenguaje codificado de un usuario "experto" en el caso de una entrevista..." (FMI, 24/09/98).

El debate ontológico en relación con la disciplina, parece demasiado consensuado en el interior de la lista como para comenzar el debate. Así, ¿cuál es el nivel de las discusiones en la lista de doctorantes y jóvenes investigadores en comunicación? No lo podemos negar, en la medida en que podemos corroborar a partir de una revisión del número de ocurrencias en el corpus ( $c f$. Figura 3, con más de 40\% de mensajes que lo mencionan): Internet, la problemática multimedia, las NTIC, las redes, la Web y todos los temas que destacan lo cyber, constituyen las temáticas más desarrolladas hasta el momento.

Es un hecho que esta pasión no es exclusiva de la lista, así como tampoco las características de los puestos ofrecidos para la contratación de jóvenes profesores. Estos hechos muestran de manera clara una imagen del mundo multimedia en la Universidad (avisos que se publican en el Journal officiel). Sin embargo, no deja de ser sorprendente. Interrogado sobre este tema, uno de los miembros de la lista proponía que el soporte electrónico de la lista lo podía explicar; incluso antes de acordar si eso se pudiese comprobar, ya no habría una lista sobre la literatura e incluso tampoco sobre sociología. En resumen, este tema se ha discutido ampliamente en la SICliste porque se trata de uno de los temas 
más discutidos por el momento. Algunas veces, las discusiones adquieren un tono casi esotérico porque en ellas se habla tanto de comunidades virtuales como de micronaciones, de bibliometría electrónica como de net-economía, de Wolton como de Mattelart, de cyber-democracia como también de cyber-vida asociativa, de cyber-televisión y de cyber-ciudadanía, de bio-juegos, de cyberperiódicos, de cyber-usos, etcétera.

"Internet" es el tema omnipresente y, sin embargo, no constituye un freno para los intercambios sobre otros temas. Los temas secundarios contienen un buen número de aspectos diversos: de los problemas metodológicos y organizacionales a preguntas de fondo ligadas muy de cerca a la investigación en comunicación, pasando por largas interrogantes acerca de temas contemporáneos. Las discusiones de fondo, de las cuales algunas son muy animadas, surgen en diversas ocasiones y nacen de forma espontánea, aun y cuando en ciertos momentos los debates sean provocados de forma explícita. Estos temas se nutren de reflexiones sobre aspectos abordados en coloquios, seminarios, jornadas de estudio que generan reacciones entre los participantes, que generan tomas de posición de algunos de los participantes acerca de algún tema de actualidad, como por ejemplo, interrogantes más específicas sobre el uso de las nuevas tecnologías. En cambio, los medios considerados en sí mismos raramente generan tomas importantes de posición.

Por último, no sería justo terminar esta rápida exploración de contenidos de los intercambios sin hablar del conjunto de preguntas prácticas planteadas entre los participantes de las SIClistes: se discute mucho sobre el propio funcionamiento de la lista (la convención, la eventual creación de un sitio Web, el acceso a los archivos) así como informaciones vitales para todo doctorante en comunicación. En este aspecto, surgen becas, premios y subvenciones, publicaciones, bibliografías, estancias académicas, formación, foros de empleo, asociaciones de doctorantes, difusión de direcciones electrónicas. En fin, temas prácticos abordados con frecuencia, como los anuncios para las jornadas de estudio, semi- 


\section{FIGURE 3}

\section{THÈMES DES INTERVENTIONS}

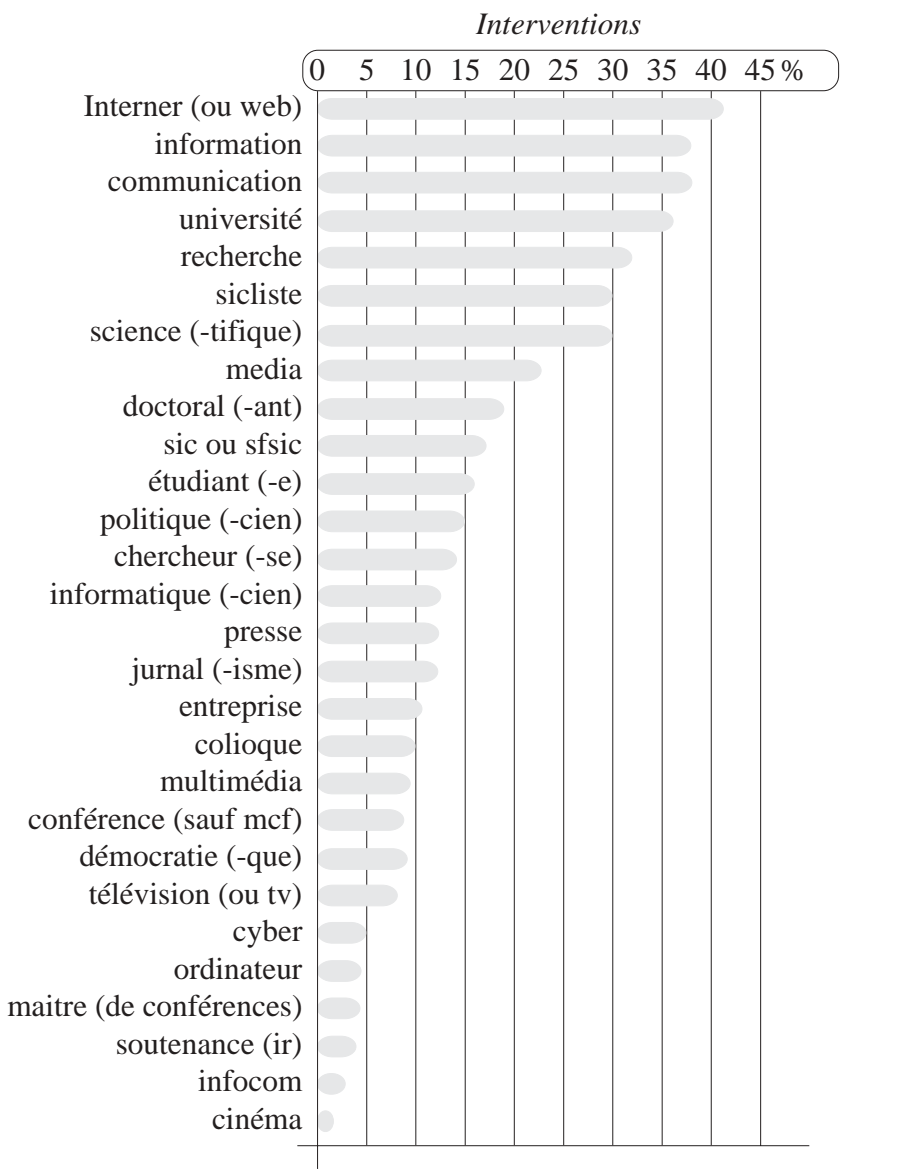

Fuente: Archives de la Sicliste. Traitement. H. Cardy \& P. Froissart, Université de parís VIII @ 2001.

narios, debates, conferencias y algunas jornadas doctorales, también recolección de artículos u ofertas de empleo en puestos universitarios o industriales, avisos de exámenes de grados y otros.

En conclusión, la SICliste constituye un intereseante espejo que permite enfocar la comunidad de jóvenes investigadores y 
doctorantes en comunicación francoparlantes. A la par de la lista oficial de la SFSIC, AgorasFSIC propone una información similar pero es más corporatista (dado que se encuentra más ligada a la vida de la Asociación), la SICliste ofrece un espacio propio sin el peso de la institución. De esta forma, las informaciones circulan más y el reservorio de información se encuentra así más alimentado. Es un sitio bastante reactivo que se puede considerar como un verdadero lugar de expresión. La SICliste se plantea verdaderamente como un punto de encuentro y de intercambio. Este sitio tiende a romper el aislamiento del que parecen sufrir los jóvenes investigadores. También se ha convertido en un lugar de reconocimiento del cual emerge una verdadera comunidad con problemas de carácter científico, institucional y editorial.

Traducción de Armando Zacarías Castillo 